\title{
ON ENERGY ESTIMATES FOR A LANDAU-LIFSCHITZ TYPE FUNCTIONAL IN HIGHER DIMENSIONS
}

\author{
LONGXing Qi AND YUtian LEI
}

\begin{abstract}
The authors study the asymptotic behavior of radial minimizers of an energy functional associated with ferromagnets and antiferromagnets in higher dimensions. The location of the zeros of the radial minimizer is discussed. Moreover, several uniform estimates for the radial minimizer are presented. Based on these estimates, the authors establish global convergence of radial minimizers.
\end{abstract}

\section{Introduction}

Let $B_{r}=\left\{x \in \mathbb{R}^{n} ;|x|<r\right\}(n \geq 2)$. Denote

$$
S^{n}=\left\{x \in \mathbb{R}^{n+1} ;\left(x^{1}\right)^{2}+\left(x^{2}\right)^{2}+\cdots+\left(x^{n+1}\right)^{2}=1\right\} .
$$

Consider the minimizers $u_{\varepsilon}$ of the energy functional

$$
E_{\varepsilon}\left(u, B_{1}\right)=\frac{1}{n} \int_{B_{1}}|\nabla u|^{n} d x+\frac{1}{2 \varepsilon^{n}} \int_{B_{1}}\left(u^{n+1}\right)^{2} d x,(\varepsilon>0),
$$

on the function class $W=\left\{u(x)=\left(\sin f(r) \frac{x}{|x|}, \cos f(r)\right) \in W^{1, n}\left(B_{1}, S^{n}\right)\right.$; $\left.f(1)=\frac{\pi}{2}, r=|x|\right\}$. Sometimes we write

$$
u_{\varepsilon}=\left(u_{\varepsilon}^{1}, u_{\varepsilon}^{2}, \ldots, u_{\varepsilon}^{n}, u_{\varepsilon}^{n+1}\right)=\left(u_{\varepsilon}^{\prime}, u_{\varepsilon}^{n+1}\right) .
$$

In the case of $n=2$, the functional $E_{\varepsilon}(u, B)$ was the Landau-Lifschitz type introduced in the study of some simplified model of high-energy physics, which controls the statics of planar ferromagnets and antiferromagnets (see $[3,7]$ ). The asymptotic behavior of minimizers of $E_{\varepsilon}\left(u, B_{1}\right)$ has been considered in [3]. In particular, they discussed the asymptotic behavior of the radial minimizer of $E_{\varepsilon}(u, B)$ in $\S 5$. When the penalization term $\frac{1}{2 \varepsilon^{2}} \int_{B_{1}}\left(u^{3}\right)^{2} d x$ is replaced by $\frac{1}{4 \varepsilon^{2}} \int_{B_{1}}\left(1-|u|^{2}\right)^{2} d x$ and $S^{2}$ is replaced by $\mathbb{R}^{2}$, the functional becomes the wellknown Ginzburg-Landau energy introduced in the theory of superconductors

Received February 24, 2008; Revised November 24, 2008.

2000 Mathematics Subject Classification. 35B25, 35J70.

Key words and phrases. radial minimizer, energy functional of Landau-Lifschitz type, planar ferromagnets and antiferromagnets, higher dimensions, location of zeros.

The research was supported partly by NSF (No.10871097) of China and Natural Science Foundation of Jiangsu Higher Education Institutions (No.08KJB110005). 
(cf. [1] and the references therein). 19 open problems were proposed in [1]. P. Mironescu studied problem 7 in $[2,6]$. Afterwards, the results were extended to the higher dimensions (cf. [5, Theorem 1.2]). In this paper, we will discuss this problem for the radial minimizer of the energy functional $E_{\varepsilon}\left(u, B_{1}\right)$ when the dimension $n \geq 2$.

Similar to the argument of Remark 3 in $[5, \S 3]$, we also have $f \in C[0,1]$ and $f(0)=0$ as long as $u \in W$. Observing the functional $E_{\varepsilon}\left(u, B_{1}\right)$, we can assume $f \in\left[0, \frac{\pi}{2}\right]$ for simplicity. First we will verify in $\S 2$ that the zeros of $u_{\varepsilon}^{\prime}$ are located near the origin. Next, as in $[5, \S 3]$, we will also consider whether

$$
\begin{aligned}
& A_{\varepsilon}=\int_{B_{1}}\left(1-\left|u_{\varepsilon}^{\prime}\right|\right)^{\alpha}\left|\nabla u_{\varepsilon}^{\prime}\right|^{n} d x \\
& B_{\varepsilon}=\int_{B_{1}}\left(1-\left|u_{\varepsilon}^{\prime}\right|\right)^{\alpha}\left|u_{\varepsilon}^{\prime}\right|^{\alpha} \mid \nabla \frac{u_{\varepsilon}^{\prime}}{\left|u_{\varepsilon}^{\prime}\right|^{n}} d x \\
& C_{\varepsilon}=\int_{B_{1}}\left|\operatorname{det}\left(\nabla u_{\varepsilon}^{\prime}\right)\right| d x
\end{aligned}
$$

have uniform upper estimates for any $\alpha>0$. We will prove in $\S 3$ the following:

Theorem 1.1. Assume $u_{\varepsilon}$ is a radial minimizer of $E_{\varepsilon}\left(u, B_{1}\right)$. Then for any $\alpha>0$, there exists a constant $C>0$ which is independent of $\varepsilon$, such that $A_{\varepsilon} \leq C$ when $\varepsilon \rightarrow 0$.

Theorem 1.2. Assume $u_{\varepsilon}$ is a radial minimizer of $E_{\varepsilon}\left(u, B_{1}\right)$. Then for any $\alpha \geq 1$, there exists a constant $C>0$ which is independent of $\varepsilon$, such that $B_{\varepsilon} \leq C$ when $\varepsilon \rightarrow 0$.

Theorem 1.3. Assume $u_{\varepsilon}$ is a radial minimizer of $E_{\varepsilon}\left(u, B_{1}\right)$. Then there exists a constant $C>0$ which is independent of $\varepsilon$, such that $C_{\varepsilon} \leq C$ when $\varepsilon \rightarrow 0$.

According to Theorem 1.2 in [4], it is not difficult to obtain the local convergence:

$$
\lim _{\varepsilon \rightarrow 0} u_{\varepsilon}(x)=\left(\frac{x}{|x|}, 0\right) \text { in } \quad W_{l o c}^{1, n}\left(B_{1} \backslash\{0\}\right) .
$$

We will set up the global convergence based on the uniform estimates in Theorems 1.1, 1.2 and 1.3.

Theorem 1.4. Assume $u_{\varepsilon}$ is a radial minimizer of $E_{\varepsilon}\left(u, B_{1}\right)$. Then we can find positive constants $L_{1}, L_{2}, L_{3}$ which are independent of $\varepsilon$, such that as $\varepsilon \rightarrow$ 0 ,

$$
\begin{gathered}
\left(1-\left|u_{\varepsilon}^{\prime}\right|\right)^{\alpha}\left|\nabla u_{\varepsilon}^{\prime}\right|^{n} \rightarrow L_{1} \delta_{o}, \quad \text { weakly star in } C\left(\overline{B_{1}}\right), \quad \forall \alpha>0, \\
\left(1-\left|u_{\varepsilon}^{\prime}\right|\right)^{\alpha}\left|u_{\varepsilon}^{\prime}\right|^{\alpha}\left|\nabla \frac{u_{\varepsilon}^{\prime}}{\left|u_{\varepsilon}^{\prime}\right|}\right|^{n} \rightarrow L_{2} \delta_{o}, \quad \text { weakly star in } C\left(\overline{B_{1}}\right), \quad \forall \alpha \geq 1, \\
\left|\operatorname{det}\left(\nabla u_{\varepsilon}\right)\right| \rightarrow L_{3} \delta_{o}, \quad \text { weakly star in } C\left(\overline{B_{1}}\right),
\end{gathered}
$$

where $\delta_{o}$ is the Dirac mass at the origin 0 . 


\section{Location of zeros}

From the direct method in the calculus of variations, it is easy to get:

Lemma 2.1. The radial minimizer $u_{\varepsilon} \in W$ satisfies

$$
-\operatorname{div}\left(|\nabla u|^{n-2} \nabla u\right)=u|\nabla u|^{n}+\frac{1}{\varepsilon^{n}}\left[u\left(u^{n+1}\right)^{2}-u^{n+1} e_{n+1}\right] \text { in } B_{1},
$$

in the weak sense, where $e_{n+1}=(0,0, \ldots, 0,1)$.

Lemma 2.2. Assume $u_{\varepsilon}$ is a radial minimizer of $E_{\varepsilon}\left(u, B_{1}\right)$. Then there exists a constant $C>0$ which is independent of $\varepsilon$, such that

$$
E_{\varepsilon}\left(u, B_{1}\right) \leq \frac{1}{n}(n-1)^{\frac{n}{2}}\left|S^{n-1}\right||\ln \varepsilon|+C .
$$

Proof. Set

$$
I(\varepsilon, R)=\min \left\{\int_{B_{R}}\left[\frac{1}{n}|\nabla u|^{n}+\frac{1}{2 \varepsilon^{n}}\left(u^{n+1}\right)^{2}\right] d x ; u_{\varepsilon} \in W^{1, n}\left(B_{R}, S^{n}\right)\right\} .
$$

Then,

$$
\begin{aligned}
I(\varepsilon, 1) & =E_{\varepsilon}\left(u_{\varepsilon}, B_{1}\right)=\frac{1}{n} \int_{B_{1}}\left|\nabla u_{\varepsilon}\right|^{n} d x+\frac{1}{2 \varepsilon^{n}} \int_{B_{1}}\left(u_{\varepsilon}^{n+1}\right)^{2} d x \\
& =\frac{1}{n} \int_{B_{\varepsilon}-1}\left|\nabla u_{\varepsilon}\right|^{n} d y+\frac{1}{2} \int_{B_{\varepsilon^{-1}}}\left(u_{\varepsilon}^{n+1}\right)^{2} d y=I\left(1, \varepsilon^{-1}\right) .
\end{aligned}
$$

Assume $u_{1}$ is a solution to $I(1,1)$. Define

$$
u_{2}=u_{1}, x \in B_{1} ; u_{2}=\frac{x}{|x|}, x \in B_{\varepsilon^{-1}} \backslash B_{1} .
$$

Since $u_{\varepsilon}$ is a minimizer, we have $E_{\varepsilon}\left(u_{\varepsilon}, B_{1}\right) \leq E_{\varepsilon}\left(u_{2}, B_{1}\right)$. Then

$$
\begin{aligned}
I\left(1, \varepsilon^{-1}\right) & \leq \frac{1}{n} \int_{B_{\varepsilon^{-1}}}\left|\nabla u_{2}\right|^{n} d x+\frac{1}{2} \int_{B_{\varepsilon^{-1}}}\left(u_{2}^{n+1}\right)^{2} d x \\
& =\frac{1}{n} \int_{B_{1}}\left|\nabla u_{1}\right|^{n} d x+\frac{1}{n} \int_{B_{\varepsilon^{-1}} \backslash B_{1}}\left|\nabla \frac{x}{|x|}\right|^{n} d x+\frac{1}{2} \int_{B_{1}}\left(u_{1}^{n+1}\right)^{2} d x \\
& =I(1,1)+\frac{1}{n}(n-1)^{\frac{n}{2}}\left|S^{n-1}\right| \int_{1}^{\varepsilon^{-1}} \frac{1}{r} d r \\
& =I(1,1)+\frac{1}{n}(n-1)^{\frac{n}{2}}\left|S^{n-1}\right||\ln \varepsilon| \\
& \leq \frac{1}{n}(n-1)^{\frac{n}{2}}\left|S^{n-1}\right||\ln \varepsilon|+C .
\end{aligned}
$$

Substituting this into $I(\varepsilon, 1)=I\left(1, \varepsilon^{-1}\right)$, we have $(2.2)$.

Lemma 2.3. Assume $\varepsilon=\varepsilon_{k}$ is a subsequence which converges to 0 , and $u_{\varepsilon}$ is a radial minimizer of $E_{\varepsilon}\left(u, B_{1}\right)$. Then there exist a positive constant $C$ independent of $\varepsilon \in(0,1)$, and a natural number $k_{0}$, such that

$$
\frac{1}{\varepsilon_{k}^{n}} \int_{B_{1}}\left(u^{n+1}\right)^{2} d x \leq C,
$$


when $k>k_{0}$.

Proof. We use the idea in [8]. Denote $V(\varepsilon)=\inf E_{\varepsilon}\left(u, B_{1}\right), u \in W$. For fixed $u \in W$, the map $\varepsilon \rightarrow E_{\varepsilon}\left(u, B_{1}\right)$ is not increasing, and

$$
\frac{\partial}{\partial \varepsilon} E_{\varepsilon}\left(u, B_{1}\right)=-\frac{n}{2 \varepsilon^{n+1}} \int_{B_{1}}\left(u^{n+1}\right)^{2} d x .
$$

Since $V(\varepsilon+\delta) \leq E_{\varepsilon+\delta}\left(u, B_{1}\right) \leq E_{\varepsilon}\left(u, B_{1}\right)=V(\varepsilon)$ for the minimizer $u=u_{\varepsilon}$ of $E_{\varepsilon}\left(u, B_{1}\right)$,

$$
\begin{aligned}
& \frac{n}{2 \varepsilon^{n+1}} \int_{B_{1}}\left(u^{n+1}\right)^{2} d x \\
= & \lim _{\delta \rightarrow 0} \frac{E_{\varepsilon}\left(u, B_{1}\right)-E_{\varepsilon+\delta}\left(u, B_{1}\right)}{\delta} \\
\leq & \varlimsup_{\delta \rightarrow 0} \frac{V(\varepsilon)-V(\varepsilon+\delta)}{\delta} \\
= & -V^{\prime}(\varepsilon) .
\end{aligned}
$$

We claim that there exists a subsequence of $\varepsilon_{k}$, which is still written as $\varepsilon_{k}$, such that

$$
-\varepsilon_{k} V^{\prime}\left(\varepsilon_{k}\right) \leq M\left(\varepsilon_{k} \rightarrow 0\right)
$$

where $M \geq \frac{1}{n}(n-1)^{\frac{n}{2}}\left|S^{n-1}\right|$. Otherwise, we suppose that there exists $\varepsilon_{0}>0$ such that $-V^{\prime}(\varepsilon)>\frac{M}{\varepsilon}$. Integrating over $\left(\varepsilon, \varepsilon_{0}\right)$, we obtain

$$
V(\varepsilon) \geq V\left(\varepsilon_{0}\right)-\int_{\varepsilon}^{\varepsilon_{0}} V^{\prime}(\varepsilon) d x>M|\ln \varepsilon|-C,
$$

which contradicts (2.2) as long as $\varepsilon$ is small enough.

Lemma 2.4. Assume $u_{\varepsilon}$ is a radial minimizer of $E_{\varepsilon}\left(u, B_{1}\right)$. Then there exist positive constants $\lambda, \mu$ which are independent of $\varepsilon \in(0,1)$, such that if

$$
\frac{1}{\varepsilon^{n}} \int_{B_{1} \cap B_{2 l \varepsilon}}\left(u_{\varepsilon}^{n+1}\right)^{2} d x \leq \mu,
$$

where $B_{2 l \varepsilon}$ is some ball of radius $2 l \varepsilon$ with $l>\lambda$, then

$$
\left|u_{\varepsilon}^{\prime}(x)\right| \geq \frac{1}{2}, \forall x \in B_{1} \cap B_{l \varepsilon} .
$$

Proof. We use the idea in [1]. First, we observe that there exists a constant $C_{2}>0$, such that for any $x \in B_{1},\left|B_{1} \cap B(x, r)\right| \geq C_{2} r^{n}$. To prove the conclusion, we will use a consequence in $[4, \S 2]$. Choose $\lambda=\frac{1}{4 C_{1}}, \mu=\frac{C_{2}}{16} \lambda^{n}$, where $C_{1}$ is the constant in (2.11) of [4]. Suppose that there is a point $x_{0} \in$ $B_{1} \cap B_{l \varepsilon}$, such that $\left|u_{\varepsilon}^{\prime}\left(x_{0}\right)\right|<\frac{1}{2}$. By (2.11) in [4], $\forall x \in B\left(x_{0}, \lambda \varepsilon\right)$,

$$
\left|u_{\varepsilon}^{\prime}(x)-u_{\varepsilon}^{\prime}\left(x_{0}\right)\right| \leq C_{1} \varepsilon^{-1}\left|x-x_{0}\right| \leq C_{1} \varepsilon^{-1}(\lambda \varepsilon)=C_{1} \lambda=\frac{1}{4} .
$$


Hence $\left(u_{\varepsilon}^{n+1}\right)^{2}=1-\left(u_{\varepsilon}^{\prime}\right)^{2}>\frac{1}{16} \forall x \in B\left(x_{0}, \lambda \varepsilon\right)$, and

$$
\int_{B_{1} \cap B\left(x_{0}, \lambda \varepsilon\right)}\left(u_{\varepsilon}^{n+1}\right)^{2} d x>\frac{1}{16}\left|B_{1} \cap B\left(x_{0}, \lambda \varepsilon\right)\right|>C_{2} \times \frac{1}{16} \times(\lambda \varepsilon)^{n}=\mu \varepsilon^{n} .
$$

Since $x_{0} \in B_{1} \cap B_{l \varepsilon}$ and $\left(B_{1} \cap B\left(x_{0}, \lambda \varepsilon\right)\right) \subset\left(B_{1} \cap B_{2 l \varepsilon}\right),(2.5)$ implies

$$
\frac{1}{\varepsilon^{n}} \int_{B_{1} \cap B_{2 l \varepsilon}}\left(u_{\varepsilon}^{n+1}\right)^{2} d x>\mu,
$$

which contradicts (2.4) and thus Lemma 2.4 is proved.

Let $u_{\varepsilon}$ be a radial minimizer of $E_{\varepsilon}\left(u, B_{1}\right) . \quad \alpha$ and $\mu$ are the constants in Lemma 2.4. If

$$
\frac{1}{\varepsilon^{n}} \int_{B_{1} \cap B_{2 \lambda \varepsilon}}\left(u_{\varepsilon}^{n+1}\right)^{2} d x \leq \mu,
$$

then $B\left(x^{\varepsilon}, \lambda \varepsilon\right)$ is called a good ball. Otherwise $B\left(x^{\varepsilon}, \lambda \varepsilon\right)$ is called a bad ball.

Now suppose that $B\left(x_{i}^{\varepsilon}, \lambda \varepsilon\right) ; i \in I$ is a family of balls satisfying

$$
\begin{aligned}
& \text { (i) } x_{i}^{\varepsilon} \in B_{1}, i \in I ; \quad \text { (ii) } B_{1} \subset \cup_{i \in I} B\left(x_{i}^{\varepsilon}, \lambda \varepsilon\right) \text {; } \\
& \text { (iii) } B\left(x_{i}^{\varepsilon}, \frac{\lambda}{4} \varepsilon\right) \cap B\left(x_{j}^{\varepsilon}, \frac{\lambda}{4} \varepsilon\right)=\emptyset, i \neq j .
\end{aligned}
$$

Denote $J_{\varepsilon}=\left\{i \in I ; B\left(x_{i}^{\varepsilon}, \lambda \varepsilon\right)\right.$ is a bad ball $\}$.

Lemma 2.5. There exists a positive integer $N$ independent of $\varepsilon \in(0,1)$, such that the number of bad balls $\operatorname{Card} J_{\varepsilon} \leq N$.

Proof. In fact, (2.6) implies that every point in $B_{1}$ can be covered by finite, say $m$ (independent of $\varepsilon$ ) balls. From (2.3) and the definition of bad balls, we have

$$
\begin{aligned}
\mu \varepsilon^{n} \operatorname{Card} J_{\varepsilon} & \leq \sum_{i \in J_{\varepsilon}} \int_{B_{1} \cap B\left(x_{i}^{\varepsilon}, 2 \lambda \varepsilon\right)}\left(u_{\varepsilon}^{n+1}\right)^{2} d x \\
& \leq m \int_{\cup_{i \in J_{\varepsilon}} B_{1} \cap B\left(x_{i}^{\varepsilon}, 2 \lambda \varepsilon\right)}\left(u_{\varepsilon}^{n+1}\right)^{2} d x \\
& \leq m \int_{B_{1}}\left(u_{\varepsilon}^{n+1}\right)^{2} d x \\
& \leq m C \varepsilon^{n}
\end{aligned}
$$

and hence $\operatorname{Card} J_{\varepsilon} \leq \frac{m C}{\mu} \leq N$.

Similar to the argument of [1, Theorem IV.1], based on Lemma 2.5 we also have the following lemma:

Lemma 2.6. There exist a subset $J \subset J_{\varepsilon}$ and a constant $h \geq \lambda$, such that

$$
\bigcup_{i \in J_{\varepsilon}} B\left(x_{i}^{\varepsilon}, \lambda \varepsilon\right) \subset \bigcup_{i \in J} B\left(x_{j}^{\varepsilon}, h \varepsilon\right)\left|x_{i}^{\varepsilon}-x_{j}^{\varepsilon}\right|>8 h \varepsilon, i, j \in J, i \neq j .
$$


Applying Lemma 2.6, we may modify the family of bad balls such that the new one, denoted by $B\left(x_{i}^{\varepsilon}, h \varepsilon\right) ; i \in J$, satisfies

$$
\begin{aligned}
& \bigcup_{i \in J_{\varepsilon}} B\left(x_{i}^{\varepsilon}, \lambda \varepsilon\right) \subset \bigcup_{i \in J} B\left(x_{j}^{\varepsilon}, h \varepsilon\right), \\
& \operatorname{Card} J \leq \operatorname{Card} J_{\varepsilon}, \\
& \left|x_{i}^{\varepsilon}-x_{j}^{\varepsilon}\right|>8 h \varepsilon, i, j \in J, i \neq j .
\end{aligned}
$$

The last condition implies that every two balls in the new family are not intersected.

Theorem 2.7. Let $u_{\varepsilon}$ be a radial minimizer of $E_{\varepsilon}\left(u, B_{1}\right)$. Then there exists a $h>0$ independent of $\varepsilon \in(0,1)$, such that

$$
Z_{\varepsilon}=\left\{x \in B_{1} ;\left|u_{\varepsilon}^{\prime}(x)\right|<\frac{1}{2}\right\} \subset B_{h \varepsilon} .
$$

Proof. Suppose there exists a point $x_{0} \in Z_{\varepsilon}$ such that $x_{0} \notin B_{h \varepsilon}$. Then all points on the circle $S_{0}=\left\{x \in B_{1} ;|x|=\left|x_{0}\right|\right\}$ satisfy $\left|u_{\varepsilon}^{\prime}(x)\right|<\frac{1}{2}$, and hence by virtue of Lemma 2.4 and (2.6), all points on $S_{0}$ are contained in bad balls. On the other hand, since $\left|x_{0}\right| \geq h \varepsilon, S_{0}$ cannot be covered by a single bad ball, i.e., $S_{0}$ is covered by at least two bad balls (which are not intersected). However, this is impossible. Theorem 2.7 is proved.

This theorem means that the zeros of $u_{\varepsilon}$ are contained in $B_{h \varepsilon}$. When $\varepsilon \rightarrow 0$, the zeros converge to the origin 0 .

\section{Proof of theorems}

Lemma 3.1. Let $R \in\left(\frac{1}{3}, \frac{1}{2}\right)$. Then there exists a constant $C>0$ independent of $\varepsilon$, such that

$$
\int_{B_{R} \backslash B_{h \varepsilon}}\left|\nabla \frac{x}{|x|}\right|^{n} d x \geq(n-1)^{\frac{n}{2}}\left|S^{n-1}\right||\ln \varepsilon|-C,
$$

when $\varepsilon$ is sufficiently small.

Proof. Clearly,

$$
\int_{B_{R} \backslash B_{h \varepsilon}}\left|\nabla \frac{x}{|x|}\right|^{n} d x=(n-1)^{\frac{n}{2}}\left|S^{n-1}\right| \int_{h \varepsilon}^{R} \frac{1}{r} d r \geq(n-1)^{\frac{n}{2}}\left|S^{n-1}\right||\ln \varepsilon|-C .
$$

Lemma 3.2. For any $\alpha>0$, there exists a constant $C>0$ independent of $\varepsilon$, such that

$$
\int_{B_{R} \backslash B_{h \varepsilon}}(1-\sin f)^{\alpha}\left|\nabla \frac{x}{|x|}\right|^{n} d x \leq C .
$$

Proof. For $\forall \alpha>0$, we choose $q=\frac{1}{\alpha}$. Applying (2.3), we obtain

$$
\frac{1}{\varepsilon^{n}} \int_{0}^{1}\left(1-\sin ^{2} f\right)^{q \alpha} r^{n-1} d r \leq \frac{1}{\varepsilon^{n}} \int_{0}^{1}\left(\cos ^{2} f\right) r^{n-1} d r \leq C .
$$


When $\alpha \in(0,1)$, we can deduce from (2.3) that

$$
\begin{aligned}
& \int_{B_{R} \backslash B_{h \varepsilon}}(1-\sin f)^{\alpha}\left|\nabla \frac{x}{|x|}\right|^{n} d x \\
\leq & C \int_{h \varepsilon}^{R}(1-\sin f)^{\alpha} \frac{r^{n-1}}{r^{n}} d r \\
\leq & C\left[\frac{1}{\varepsilon^{n}} \int_{0}^{1}\left(1-\sin ^{2} f\right)^{q \alpha} r^{n-1} d r\right]^{\frac{1}{q}} \varepsilon^{\frac{n}{q}}\left[\int_{h \varepsilon}^{R} r^{\frac{q+n-1}{1-q}} d r\right]^{1-\frac{1}{q}} \\
\leq & C \varepsilon^{\frac{n}{q}}\left[\varepsilon^{\frac{n}{1-q}}-C(R)\right]^{1-\frac{1}{q}} \\
\leq & C \varepsilon^{\frac{n}{q}} \varepsilon^{-\frac{n}{q}}=C .
\end{aligned}
$$

When $\alpha \geq 1$, it is easy to deduce (3.2) by the argument above.

Lemma 3.3. There exists a constant $C>0$ which is independent of $\varepsilon$, such that

$$
\int_{B_{1}} \cos ^{n} f|\nabla f|^{n} d x+\int_{B_{h \varepsilon}} \sin ^{n} f\left|\nabla \frac{x}{|x|}\right|^{n} d x+\int_{B_{1} \backslash B_{R}} \sin ^{n} f\left|\nabla \frac{x}{|x|}\right|^{n} d x \leq C .
$$

Proof. From Lemma 2.2, we have

$$
\int_{B_{1}}\left|\nabla u_{\varepsilon}^{\prime}\right|^{n} d x \leq(n-1)^{\frac{n}{2}}\left|S^{n-1}\right||\ln \varepsilon|+C .
$$

Noting

$$
\nabla u_{\varepsilon}^{\prime}=\nabla\left(\sin f \frac{x}{|x|}\right)=\nabla(\sin f) \frac{x}{|x|}+\sin f \nabla \frac{x}{|x|}=\cos f \nabla f \frac{x}{|x|}+\sin f \nabla \frac{x}{|x|} .
$$

We obtain from Jensen's inequality that

$$
\begin{aligned}
& \quad \int_{B_{1}} \cos ^{n} f|\nabla f|^{n} d x+\int_{B_{h \varepsilon}} \sin ^{n} f\left|\nabla \frac{x}{|x|}\right|^{n} d x+\int_{B_{1} \backslash B_{h \varepsilon}} \sin ^{n} f\left|\nabla \frac{x}{|x|}\right|^{n} d x \\
& \leq(n-1)^{\frac{n}{2}}\left|S^{n-1}\right||\ln \varepsilon|+C .
\end{aligned}
$$

(3.4) subtracts (3.1). Then we can deduce that

$$
\begin{aligned}
\int_{B_{1}} \cos ^{n} f|\nabla f|^{n} d x & +\int_{B_{h \varepsilon}} \sin ^{n} f\left|\nabla \frac{x}{|x|}\right|^{n} d x+\int_{B_{1} \backslash B_{R}} \sin ^{n} f\left|\nabla \frac{x}{|x|}\right|^{n} d x \\
& -\int_{B_{R} \backslash B_{h \varepsilon}}\left(1-\sin ^{n} f\right)\left|\nabla \frac{x}{|x|}\right|^{n} d x \leq C .
\end{aligned}
$$

Using Lemma 3.2, we get

$$
\int_{B_{1}} \cos ^{n} f|\nabla f|^{n} d x+\int_{B_{h \varepsilon}} \sin ^{n} f\left|\nabla \frac{x}{|x|}\right|^{n} d x+\int_{B_{1} \backslash B_{R}} \sin ^{n} f\left|\nabla \frac{x}{|x|}\right|^{n} d x \leq C .
$$


Proof of Theorem 1.1. From Lemma 3.3, we deduce that

$$
\begin{aligned}
& \int_{\left(B_{1} \backslash B_{R}\right) \cup B_{h \varepsilon}}\left(1-\left|u_{\varepsilon}^{\prime}\right|\right)^{\alpha}\left|\nabla u_{\varepsilon}^{\prime}\right|^{n} d x \\
= & \int_{\left(B_{1} \backslash B_{R}\right) \cup B_{h \varepsilon}}(1-\sin f)^{\alpha}\left|\nabla\left(\sin f \frac{x}{|x|}\right)\right|^{n} d x \\
\leq & \int_{\left(B_{1} \backslash B_{R}\right) \cup B_{h \varepsilon}}\left|\nabla(\sin f) \frac{x}{|x|}+\sin f \nabla \frac{x}{|x|}\right|^{n} d x \\
\leq & C \int_{\left(B_{1} \backslash B_{R}\right) \cup B_{h \varepsilon}}\left(\cos ^{n} f|\nabla f|^{n}+\sin ^{n} f\left|\nabla \frac{x}{|x|}\right|^{n}\right) d x \\
\leq & C\left(\int_{B_{1}} \cos ^{n} f|\nabla f|^{n} d x+\int_{B_{h \varepsilon}} \sin ^{n} f\left|\nabla \frac{x}{|x|}\right|^{n} d x+\int_{B_{1} \backslash B_{R}} \sin ^{n} f\left|\nabla \frac{x}{|x|}\right|^{n} d x\right) \\
\leq & C .
\end{aligned}
$$

By Lemma 3.2 and Lemma 3.3, we obtain

$$
\begin{aligned}
& \int_{B_{R} \backslash B_{h \varepsilon}}\left(1-\left|u_{\varepsilon}^{\prime}\right|\right)^{\alpha}\left|\nabla u_{\varepsilon}^{\prime}\right|^{n} d x \\
(3.6)= & \int_{B_{R} \backslash B_{h \varepsilon}}(1-\sin f)^{\alpha}\left|\nabla\left(\sin f \frac{x}{|x|}\right)\right|^{n} d x \\
\leq & C\left(\int_{B_{R} \backslash B_{h \varepsilon}} \cos ^{n} f|\nabla f|^{n} d x+\int_{B_{R} \backslash B_{h \varepsilon}}(1-\sin f)^{\alpha}\left|\nabla \frac{x}{|x|}\right|^{n} d x\right) \leq C .
\end{aligned}
$$

Combining (3.5) with (3.6) yields

$$
A_{\varepsilon}=\int_{B_{1}}\left(1-\left|u_{\varepsilon}^{\prime}\right|\right)^{\alpha}\left|\nabla u_{\varepsilon}^{\prime}\right|^{n} d x \leq C .
$$

Theorem 1.1 is complete.

Proof of Theorem 1.2. Obviously,

$$
\begin{aligned}
B_{\varepsilon} & =\int_{B_{1}}\left(1-\left|u_{\varepsilon}^{\prime}\right|\right)^{\alpha}\left|u_{\varepsilon}^{\prime}\right|^{\alpha}\left|\nabla \frac{u_{\varepsilon}^{\prime}}{\left|u_{\varepsilon}^{\prime}\right|}\right|^{2} d x=\int_{B_{1}}(1-\sin f)^{\alpha} \sin ^{\alpha} f\left|\nabla \frac{x}{|x|}\right|^{n} d x \\
& \leq \int_{B_{1}}\left(1-\sin ^{2} f\right)^{\alpha} \sin ^{\alpha} f\left|\nabla \frac{x}{|x|}\right|^{n} d x=\int_{B_{1}} \cos ^{2 \alpha} f \sin ^{\alpha} f\left|\nabla \frac{x}{|x|}\right|^{n} d x \\
& =(n-1)^{\frac{n}{2}}\left|S^{n-1}\right| \int_{0}^{1} \cos ^{2 \alpha} f \sin ^{\alpha} f \frac{1}{r} d r \\
& =(n-1)^{\frac{n}{2}}\left|S^{n-1}\right| \int_{0}^{\delta} \cos ^{2 \alpha} f \sin ^{\alpha} f \frac{1}{r} d r+(n-1)^{\frac{n}{2}}\left|S^{n-1}\right| \int_{\delta}^{1} \cos ^{2 \alpha} f \sin ^{\alpha} f \frac{1}{r} d r \\
& \leq(n-1)^{\frac{n}{2}}\left|S^{n-1}\right| \int_{0}^{\delta}\left(\cos ^{2 \alpha} f\right) f^{\alpha} \frac{1}{r} d r+C(\delta) .
\end{aligned}
$$


When $\alpha \in(1, n]$, by the mean value theorem and $f(0)=0$, there exists $\xi \in(0,1)$ such that

$$
\frac{1}{r} \sin ^{\alpha} f \leq|\cos f(\xi r)|^{\alpha}\left|f^{\prime}(\xi r)\right|^{\alpha} r^{\alpha-1} \leq C|\cos f(\xi r)|^{\alpha}|\nabla f(\xi r)|^{\alpha} r^{\alpha-1} .
$$

Thus, we have

$$
\begin{aligned}
B_{\varepsilon} & \leq(n-1)^{\frac{n}{2}}\left|S^{n-1}\right| \int_{0}^{\delta} \cos ^{2 \alpha} f|\nabla f|^{\alpha} r^{\alpha-1} d r+C \\
& \leq C\left[\int_{0}^{\delta} \cos ^{n} f(\xi r)|\nabla f(\xi r)|^{n} r^{n-1} d r\right]^{\frac{\alpha}{n}}\left[\int_{0}^{\delta} r^{\frac{\alpha-n}{n-1}} d r\right]^{\frac{n-1}{n}}+C \\
& \leq C \xi^{\frac{\alpha(1-n)}{n}}\left[\int_{0}^{\xi \delta} \cos ^{n} f|\nabla f|^{n} s^{n-1} d s\right]^{\frac{\alpha}{n}}\left(\left.\frac{n-1}{\alpha-1} r^{\frac{\alpha-1}{n-1}}\right|_{0} ^{\delta}\right)^{\frac{n-1}{n}}+C \\
& \leq C \int_{B_{1}} \cos ^{n} f|\nabla f|^{n} d x+C .
\end{aligned}
$$

From Lemma 3.3, we obtain $B_{\varepsilon} \leq C$.

When $\alpha=1$, the conclusion is easy to be obtained by the argument above. When $\alpha>n$, we can set $\alpha=n+\beta$. Thus by the mean value theorem and $f(0)=0$, there exists $\xi \in(0,1)$ such that

$$
\frac{1}{r} \sin ^{n} f \leq|\cos f(\xi r)|^{n}\left|f^{\prime}(\xi r)\right|^{n} r^{n-1} .
$$

Hence, we can deduce that, by using Lemma 3.3,

$$
\begin{aligned}
& \int_{0}^{\delta}\left(\cos ^{2 \alpha} f\right) f^{\alpha} \frac{1}{r} d r \\
\leq & \int_{0}^{\delta}\left(\cos ^{2 \alpha} f\right) f^{\beta}|\cos f(\xi r)|^{n}\left|f^{\prime}(\xi r)\right|^{n} r^{n-1} d r \\
\leq & \int_{0}^{\delta}|\cos f(\xi r)|^{n}\left|f^{\prime}(\xi r)\right|^{n} r^{n-1} d r \leq C .
\end{aligned}
$$

The rest proof is easy to be completed.

The proof of Theorem 1.3.

$$
\begin{aligned}
\operatorname{det}\left(\nabla u_{\varepsilon}^{\prime}\right)= & \left|\begin{array}{cccc}
\left(\sin f \frac{x_{1}}{|x|}\right)_{x_{1}} & \left(\sin f \frac{x_{2}}{|x|}\right)_{x_{1}} & \cdots & \left(\sin f \frac{x_{n}}{|x|}\right)_{x_{1}} \\
\left(\sin f \frac{x_{1}}{|x|}\right)_{x_{2}} & \left(\sin f \frac{x_{2}}{|x|}\right)_{x_{2}} & \cdots & \left(\sin f \frac{x_{n}}{|x|}\right)_{x_{2}} \\
\cdots & \cdots & \cdots & \cdots \\
\left(\sin f \frac{x_{1}}{|x|}\right)_{x_{n}} & \left(\sin f \frac{x_{2}}{|x|}\right)_{x_{n}} & \cdots & \left(\sin f \frac{x_{n}}{|x|}\right)_{x_{n}}
\end{array}\right| \\
= & \left|\begin{array}{cccc}
\cos f f_{x_{1}} \frac{x_{1}}{|x|}+\sin f \frac{|x|^{2}-x_{1}^{2}}{|x|^{3}} & \cos f f_{x_{1}} \frac{x_{2}}{|x|}-\sin f \frac{x_{1} x_{2}}{\mid x 3^{3}} & \cdots & \cos f f_{x_{1}} \frac{x_{n}}{|x|}-\sin f \frac{x_{1} x_{n}}{|x|^{3}} \\
\cos f f_{x_{2}} \frac{x_{1}}{|x|}-\sin f \frac{x_{1} x_{2}}{|x|^{3}} & \cos f f_{x_{2}} \frac{x_{2}}{|x|}+\sin f \frac{|x|^{2}-x_{2}^{2}}{|x|^{3}} & \cdots & \cos f f_{x_{2}} \frac{x_{n}}{|x|}-\sin f \frac{x_{2} x_{n}}{|x|^{3}} \\
\cdots & \cdots & \cdots \\
\cos f f_{x_{n}} \frac{x_{1}}{|x|}-\sin \frac{x_{1} x_{n}}{|x|^{3}} & \cos f f_{x_{n}} \frac{x_{2}}{|x|}-\sin f \frac{x_{2} x_{n}}{|x|^{3}} & \cdots & \cos f f_{x_{n}} \frac{x_{n}}{|x|}+\sin \frac{|x|^{2}-x_{n}^{2}}{|x|^{3}}
\end{array}\right| .
\end{aligned}
$$


It is easy to derive that

$$
\begin{aligned}
& \left|\operatorname{det}\left(\nabla u_{\varepsilon}^{\prime}\right)\right| \\
\leq & \cos ^{n} f|\nabla f|^{n}+\cos ^{n-1} f|\nabla f|^{n-1} \sin f \frac{1}{|x|}+\cos ^{n-2} f|\nabla f|^{n-2} \sin ^{2} f \frac{1}{|x|^{2}} \\
& +\cdots+\cos f|\nabla f| \sin ^{n-1} f \frac{1}{|x|^{n-1}}+\sin ^{n} f \frac{1}{|x|^{n}} \\
\leq & \cos ^{n} f|\nabla f|^{n}+\cos ^{n-1} f|\nabla f|^{n-1} \frac{f}{r}+\cos ^{n-2} f|\nabla f|^{n-2} \frac{f^{2}}{r^{2}} \\
& +\cdots+\cos f|\nabla f| \frac{f^{n-1}}{r^{n-1}}+\frac{f^{n}}{r^{n}} .
\end{aligned}
$$

Similar to the derivation of Theorem 1.2, using the mean value theorem, we derive

$$
\begin{aligned}
\left|\operatorname{det}\left(\nabla u_{\varepsilon}^{\prime}\right)\right| \leq & \cos ^{n} f|\nabla f|^{n}+\cos ^{n-1} f|\nabla f|^{n}+\cos ^{n-2} f|\nabla f|^{n}+\cdots+ \\
& +\cos f|\nabla f|^{n}+|\nabla f|^{n} .
\end{aligned}
$$

Noting $\cos f(0)=\cos 0=1$ and $\cos f$ is continuous near the zero, we know that there exists $\delta>0$, such that when $r \in(0, \delta)$,

$$
\begin{aligned}
|\cos f(r)-\cos f(0)| \leq \frac{1}{2} & \Rightarrow \cos f(r) \geq \cos f(0)-\frac{1}{2}=\frac{1}{2} \\
& \Rightarrow \cos ^{i} f(r) \leq C \cos ^{n} f(r), \quad i=1,2, \ldots, n-1 .
\end{aligned}
$$

From this result, (3.7) and Hölder's inequality, we can deduce that

$$
\begin{aligned}
C_{\varepsilon} & =\int_{B_{1}}\left|\operatorname{det}\left(\nabla u_{\varepsilon}^{\prime}\right)\right| d x \\
& \leq C(n, \delta) \int_{B_{1} \backslash B_{\delta}} \cos ^{n} f|\nabla f|^{n} d x+C \int_{B_{\delta}} \cos ^{n} f|\nabla f|^{n} d x \\
& \leq C+C \int_{B_{1}} \cos ^{n} f|\nabla f|^{n} d x \\
& \leq C .
\end{aligned}
$$

Theorem 1.3 is complete.

Proof of Theorem 1.4. Theorem 1.1 means that the $L^{1}\left(B_{1}\right)$-norm of

$$
\left(1-\left|u_{\varepsilon}^{\prime}\right|\right)^{\alpha}\left|\nabla u_{\varepsilon}^{\prime}\right|^{n}
$$

is bounded. Therefore, there exists a Radon measure $\mu_{1}$ such that

$$
\lim _{\varepsilon \rightarrow 0}\left(1-\left|u_{\varepsilon}^{\prime}\right|\right)^{\alpha}\left|\nabla u_{\varepsilon}^{\prime}\right|^{n}=\mu_{1} \quad \text { weakly star in } C\left(\overline{B_{1}}\right) .
$$

Similar to the derivation (3.3) in [5], from (3.3) and (2.3) it also follows

$$
\lim _{\varepsilon \rightarrow 0} \int_{B_{1} \backslash B_{R}}\left(1-\left|u_{\varepsilon}^{\prime}\right|\right)^{\alpha}\left|\nabla u_{\varepsilon}^{\prime}\right|^{n} d x=0
$$


for any $R>0$, and hence $\operatorname{supp}\left(\mu_{1}\right)=\{0\}$. Thus, we can find $L_{1} \in[0, \infty)$ such that

$$
\mu_{1}=L_{1} \delta_{o}
$$

We claim $L_{1}>0$. In fact, by virtue of $f(r) \in C[0,1]$ and $f(0)=0, f(h \varepsilon) \geq 1 / 2$ which can be seen by Theorem 2.7, there must exists $r_{\varepsilon} \in(0, h \varepsilon)$ such that $f\left(r_{\varepsilon}\right)=1 / 4$. Using (2.11) in [4], we can find a sufficiently small positive constant $\delta$ which is independent of $\varepsilon$, such that

Therefore,

$$
\frac{1}{8} \leq f(x) \leq \frac{3}{8}, \quad r \in\left(r_{\varepsilon}-\delta \varepsilon, r_{\varepsilon}+\delta \varepsilon\right)
$$

$$
\begin{aligned}
& \int_{B\left(0, r_{\varepsilon}+\delta \varepsilon\right) \backslash B\left(0, r_{\varepsilon}+\delta \varepsilon\right)}(1-\sin f)^{\alpha}\left|\nabla u_{\varepsilon}\right|^{n} d x \\
\geq & \left|S^{n-1}\right|\left(\sin \frac{1}{8}\right)^{2}\left(1-\sin \frac{3}{8}\right)^{\alpha} \int_{r_{\varepsilon}-\delta \varepsilon}^{r_{\varepsilon}+\delta \varepsilon} \frac{d r}{r}>0 .
\end{aligned}
$$

This implies $L_{1}>0$. Eq.(1.1) is proved.

By the same argument of above, (1.2) can also be verified.

Next, Theorem 1.3 means that the $L^{1}\left(B_{1}\right)$-norm of $\left|\operatorname{det}\left(\nabla u_{\varepsilon}^{\prime}\right)\right|$ is bounded. Therefore, there exists a Radon measure $\mu_{3}$ such that

$$
\lim _{\varepsilon \rightarrow 0}\left|\operatorname{det}\left(\nabla u_{\varepsilon}^{\prime}\right)\right|=\mu_{3} \quad \text { weakly star in } C\left(\overline{B_{1}}\right) \text {. }
$$

By an analogous argument of Remarks 2 and 3 in [5, pp. 131-133], we can also find $L_{3} \in(0, \infty)$ such that

$$
\mu_{3}=L_{3} \delta_{o} .
$$

Then (1.3) is proved and Theorem 1.4 is complete.

Acknowledgements. The authors would like to thank the referee(s) for the helpful comments which improve the presentation of this paper.

\section{References}

[1] F. Bethuel, H. Brezis, and F. Hélein, Ginzburg-Landau Vortices, Birkhäuser, Berlin, 1994.

[2] M. Comte and P. Mironescu, The behavior of a Ginzburg-Landau minimizer near its zeroes, Calc. Var. Partial Differential Equations 4 (1996), no. 4, 323-340.

[3] F. B. Hang and F. H. Lin, Static theory for planar ferromagnets and antiferromagnets, Acta Math. Sin. (Engl. Ser.) 17 (2001), no. 4, 541-580.

[4] Y. T. Lei, Asymptotic behavior of the regularized minimizer of an energy functional in higher dimensions, J. Math. Anal. Appl. 334 (2007), no. 2, 1341-1362.

[5] _ Some results on an n-Ginzburg-Landau-type minimizer, J. Comput. Appl. Math. 217 (2008), no. 1, 123-136.

[6] P. Mironescu, Une estimation pour les minimiseurs de l'énergie de Ginzburg-Landau, C. R. Acad. Sci. Paris Ser. I Math. 319 (1994), no. 9, 941-943.

[7] N. Papanicolaou and P. N. Spathis, Semitopological solitons in planar ferromagnets, Nonlinearity 12 (1999), no. 2, 285-302.

[8] M. Struwe, Une estimation asymptotique pour le modéle de Ginzburg-Landau, C. R. Acad. Sci. Paris Ser. I Math. 317 (1993), no. 7, 677-680. 
LONGXING QI

Institute OF Mathematics

School of Mathematics and Computer Science

NANJing Normal University

NANJing, 210097, P. R. China

Yutian Lei

Institute of Mathematics

School of Mathematics and Computer Science

NANJing NoRmal UnIVERsity

NANJing, 210097, P. R. China

E-mail address: lythxl@163.com 